\title{
CONF-950412--25
}

\section{PULSED-LASER DEPOSITION OF TITANIUM NITRIDE}

\author{
WENBIAO JIANG and M. GRANT NORTON \\ Department of Mechanical and Materials Engineering \\ Washington State University \\ Pullman Washington 99164 \\ J. THOMAS DICKINSON \\ Department of Physics \\ Washington State University \\ Pullman Washington 99164 \\ N.D. EVANS \\ Oak Ridge Institute for Science and Education \\ Oak Ridge Tennessee 37831 \\ submitted for publication in
}

\section{Film Synthesis and Growth Using Energetic Beams}

edited by H.A. Atwater, J.T. Dickinson, D.H. Lowndes, and A. Polman

Materials Research Society Symposium Proceedings

April 1995

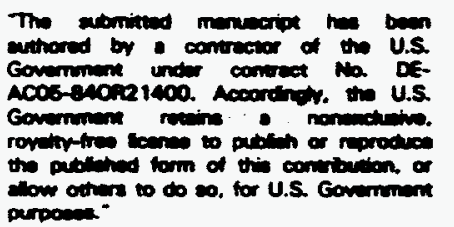




\title{
PULSED-LASER DEPOSITION OF TITANIUM NITRIDE
}

\author{
WENBIAO ЛIANG*, M. GRANT NORTON*, J. THOMAS DICKINSON**, AND \\ N.D. EVANS ${ }^{* * *}$ \\ - Department of Mechanical and Materials Engineering and \\ ** Department of Physics, Washington State University, Pullman, WA 99164 \\ *** Oak Ridge Institute for Science and Education, Oak Ridge, TN 37831
}

\section{ABSTRACT}

The pulsed-laser deposition technique has been used to form thin films of TiN on (100)oriented single crystal substrates of silicon and rocksalt. Using atomic force microscopy, it was revealed that TiN films grown on silicon at substrate temperatures ranging from $50^{\circ} \mathrm{C}$ to $500^{\circ} \mathrm{C}$ were extremely smooth-the mean roughness being $\sim 0.2 \mathrm{~nm}$. Thin TiN films deposited on freshly cleaved $\mathrm{NaCl}$ were found to be epitaxial at substrate temperatures as low as $50^{\circ} \mathrm{C}$. Epitaxy in this latter system is believed to be due to the structural similarity between film and substrate and the almost exact $4: 3$ coincident site lattice.

\section{INTRODUCTION}

With excellent mechanical, thermal, and electronic properties, such as good thermal stability, high corrosion resistance, and low electrical resistivity, titanium nitride (TiN) thin films have many applications ranging from coatings on cutting tools to diffusion barriers in VLSI microelectronics. This broad range of applications has resulted in the development of a wide variety of deposition techniques to form TiN films. These techniques include both physical and chemical vapor deposition methods. However, most of these techniques require moderate to high substrate temperatures to form oriented crystalline films. The high deposition temperature inhibits the use of TiN films in some applications where the substrate cannot withstand elevated temperatures. It is therefore of interest to study the possibility of TiN deposition at lower substrate temperatures.

The major obstacle to low temperature growth is the difficulty of obtaining the high surface mobility required for the nucleation and growth of crystalline or epitaxial films at low substrate temperatures. This limitation can be overcome by delivering the material to the substrate in unique chemical forms (e.g., clusters or reactive species), in charged or highly excited states, and/or with appreciable kinetic energy. The latter objective may be accomplished by the use of pulsed-laser deposition (PLD). In PLD, a focused high-power excimer laser is directed towards a target in a high-vacuum chamber. The strong laser-target interaction results in the formation of a high temperature, high velocity, and electronically excited plasma consisting of neutral atoms, molecules, ions, and electrons. With little loss of energy in high vacuum, the plasma is transported to and deposited onto a substrate.

Several groups have reported the formation of TiN thin films by PLD using irradiation of either metallic targets in a nitriding atmosphere $[1,2]$ or stoichiometric nitride targets $[1,3,4]$. Even when the substrate is maintained at room temperature during film deposition, crystalline TiN films have been formed on silicon [3]. Narayan and co-workers have reported the formation of single crystal TiN films on silicon substrates heated during deposition to temperatures in the range 600 $700^{\circ} \mathrm{C} \mathrm{[4].} \mathrm{This} \mathrm{latter} \mathrm{result} \mathrm{is} \mathrm{unusual} \mathrm{given} \mathrm{the} \mathrm{relatively} \mathrm{large} \mathrm{lattice} \mathrm{mismatch} \mathrm{(25 \% )} \mathrm{between}$ $\mathrm{Si}\left(\mathrm{a}_{0}=0.543 \mathrm{~nm}\right)$ and $\mathrm{TiN}\left(\mathrm{a}_{0}=0.422 \mathrm{~nm}\right)$. The cube-on-cube epitaxy appears to be enhanced by the $4: 3$ near-coincident site lattice where the mismatch is less than $4 \%$. 


\section{DISCLAIMER}

Portions of this document may be illegible in electronic image products. Images are produced from the best available original document. 
Titanium nitride films were formed by focusing a Lambda Physik EMG203 excimer laser operating on $\operatorname{KrF}(\lambda=248 \mathrm{~nm}$ ) onto a hot-pressed TiN target supplied by Target Materials Inc. The laser fluence was $4 \mathrm{~J} / \mathrm{cm}^{2}$, the pulse duration was $20 \mathrm{~ns}$, and the pulse repetition rate was in the range 4 to $8 \mathrm{~Hz}$. The films were deposited in vacuum (base pressure $10^{-6}$ torr). Two types of substrate material were used in the present study: (100)-oriented single crystal silicon and rocksalt. (No attempt was made to remove the native oxide layer on the silicon substrates.) The substrates were mechanically clamped onto the stainless steel plate of a small resistive heater and were positioned parallel to the target. The distance between the target surface and the substrate was $\sim 5 \mathrm{~cm}$. The temperature of the substrate heater was monitored by a thermocouple embedded into the heater block at a position just behind the substrate. All the temperatures reported here are those of the substrate heater as measured by the thermocouple.

The rocksalt substrates were used to facilitate sample preparation for transmission electron microscopy (TEM) and for parallel electron energy loss spectrometry (PEELS). Thin ( 10 to 30 $\mathrm{nm}$ thick) TiN films were deposited onto freshly cleaved (100)-oriented $\mathrm{NaCl}$ substrates. Following deposition, the $\mathrm{NaCl}$ was dissolved in deionized water and the film collected on a slotted 400 mesh copper grid and dried in air. This specimen preparation method produces excellent samples for PEELS analysis - they are thin and of a uniform thickness. All the films were analyzed either immediately after deposition or after being stored in a desiccator for a few days. A Hitachi H600 TEM operated at $100 \mathrm{kV}$ was used to examine the films. PEELS was performed with a Philips EM400T/FEG analytical electron microscope operated at $100 \mathrm{kV}$.

The surface morphology of the films was characterized with a Digital Instruments Nanoscope III Multimode atomic force microscope (AFM) operated in either contact mode or tapping mode. The images shown here are top view images with linear gray scale encoding of the height of features. The films were also examined using a JEOL JSM 6400 scanning electron microscope (SEM) operated at $20 \mathrm{kV}$. No conductive coating was applied to the samples prior to SEM analysis.

\section{RESULTS AND DISCUSSION}

The first obvious feature of all the films grown in this study was their golden-yellow color, which is characteristic of TiN [5]. The surfaces of the films were fairly free of the ubiquitous particulates associated with films formed by PLD. The occurrence of particulates on the film surface is highly dependent on the nature of the target [1]. TiN films formed from the ablation of titanium may have large spherical particles on the surface as a result of the ejection of molten metal from the target [2]. Films made from pre-formed TiN targets of ten show a lower number of particulates [1].

Examination of the surface morphology of the films by AFM showed that they were extremely smooth for all the substrate temperatures used in this study. Figure 1 shows AFM images of the surface of a silicon substrate and of TiN films grown at three substrate temperatures. Mean roughness values determined by the AFM software for the TiN films varied from $0.18 \mathrm{~nm}$ for films deposited at a substrate temperature of $500^{\circ} \mathrm{C}$ to $0.27 \mathrm{~nm}$ for films deposited at $50^{\circ} \mathrm{C}$. These values can be compared to the mean roughness of $0.19 \mathrm{~nm}$ determined for the uncoated silicon wafer. 


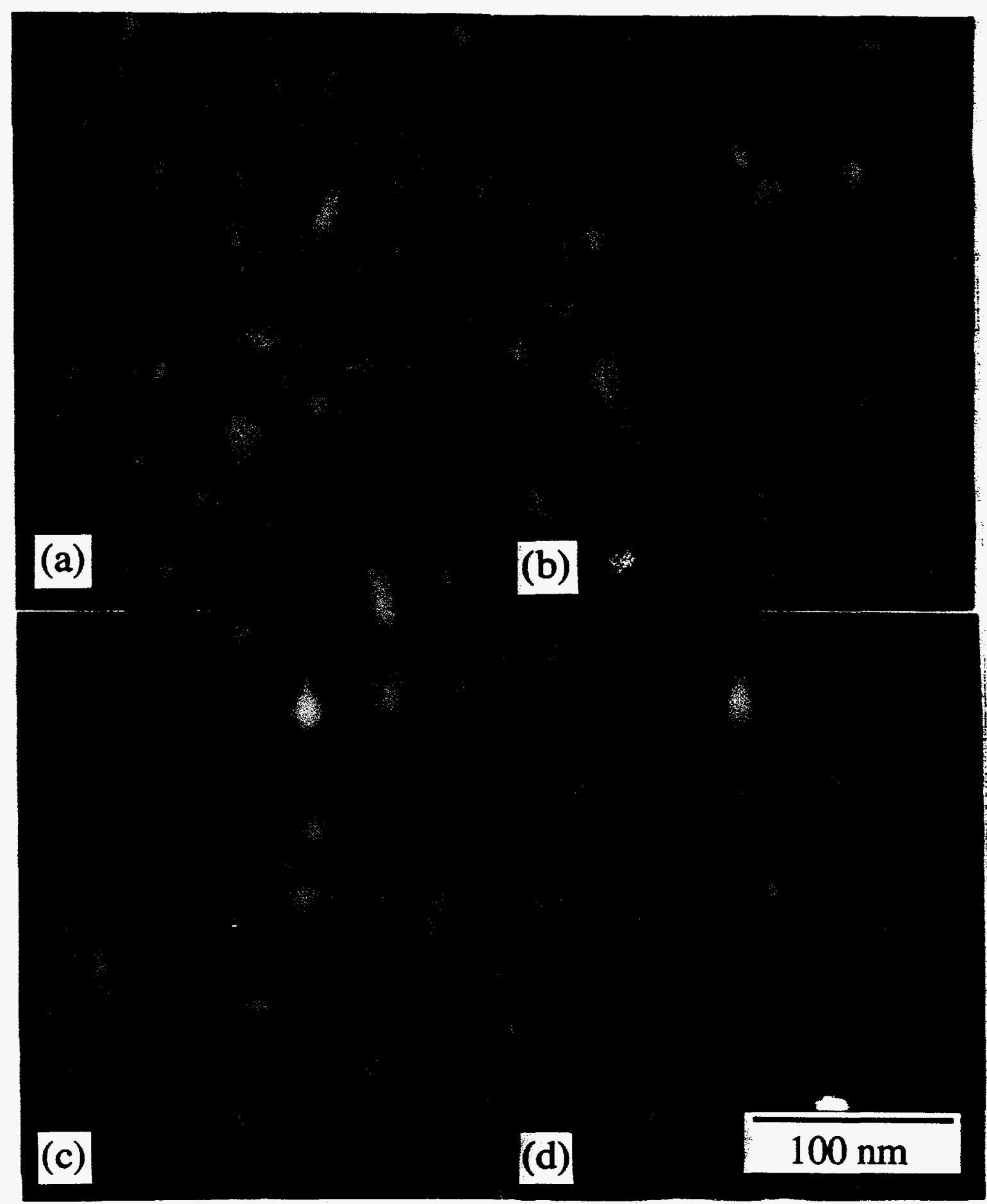

Figure 1. The surface morphology of (a) (100)-oriented silicon and (b) - (d) TiN thin films deposited onto silicon substrates at temperatures of (b) $50^{\circ} \mathrm{C}$, (c) $300^{\circ} \mathrm{C}$, and (d) $500^{\circ} \mathrm{C}$. The $\mathrm{z}$-range is $3 \mathrm{~nm}$ in all the images.

Figure 2 shows a series of X-ray diffraction patterns obtained from TiN thin films deposited on (100)-oriented silicon substrates at different temperatures. At a substrate temperature of $50^{\circ} \mathrm{C}$, no strong diffraction maxima are observed. For films deposited at substrate temperatures of $300^{\circ} \mathrm{C}$ and $500^{\circ} \mathrm{C}$ sharp peaks in the patterns corresponding to diffraction from the (200) planes of TiN are observed indicating that these films are textured. Several groups have reported that TiN 
films on silicon are oriented with the $[100]$ of TiN parallel to the $[100]$ of Si $[1,4]$. Recent results on TiN films prepared by reactive of magnetron sputtering indicate that the preferred film orientation changes from (200) to (111) with increasing film thickness [6].

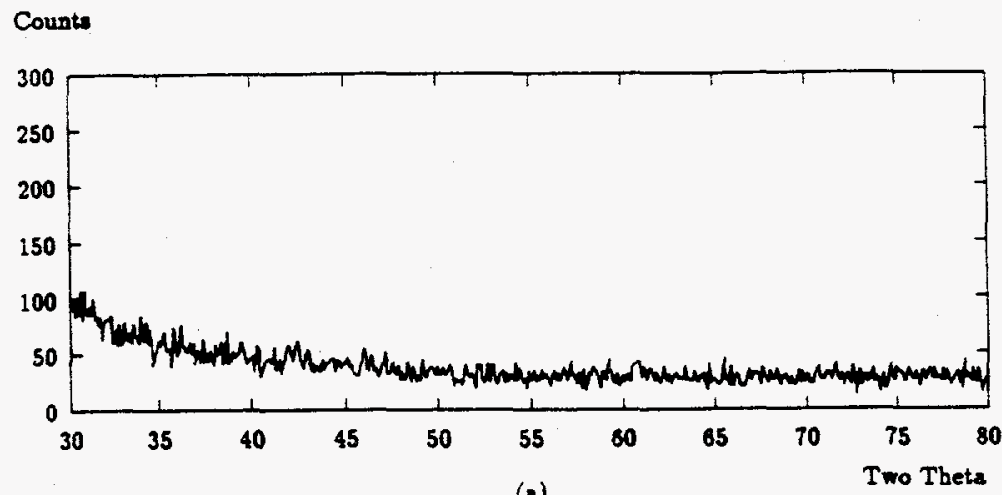

(a)

Counts

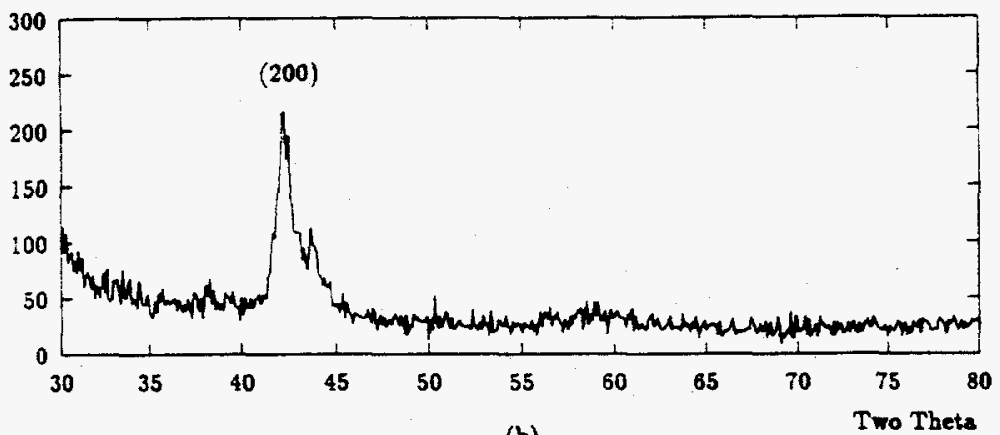

(b)

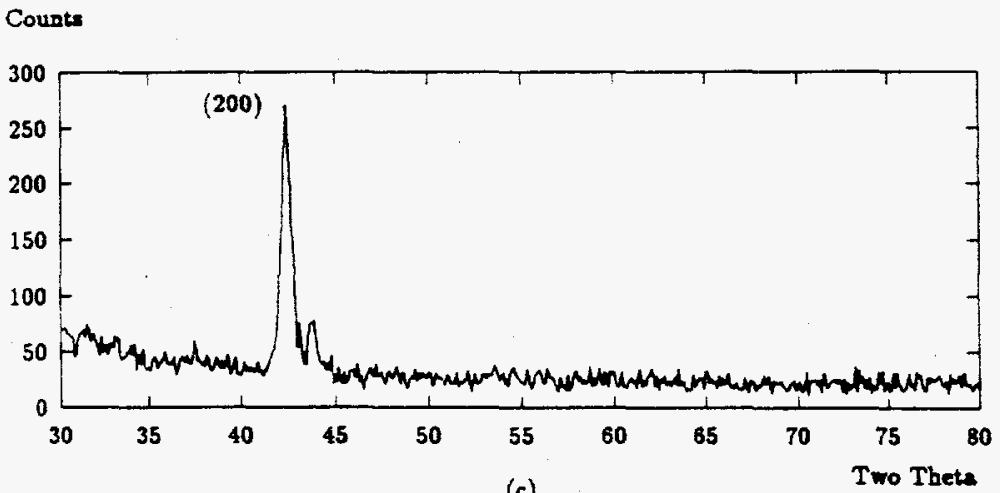

(c)

Figure 2. X-ray diffraction patterns recorded from $\mathrm{TiN}$ thin films deposited on (100)-oriented $\mathrm{Si}$ substrates at substrate temperatures of (a) $50^{\circ} \mathrm{C}$, (b) $300^{\circ} \mathrm{C}$, and (c) $500^{\circ} \mathrm{C}$.

Electron channeling patterns (ECP), shown in Figure 3, obtained from very thin TiN films deposited on silicon at a substrate temperature of $300^{\circ} \mathrm{C}$ indicate that the films are highly ordered, at this stage of growth, in the film-substrate interface plane. No clear channelling patterns were acquired from thicker TiN films deposited under equivalent conditions.

Figures $4 \mathrm{a}$ and $4 \mathrm{~b}$ show selected area electron diffraction (SAED) patterns recorded from free-standing thin TiN films deposited on $\mathrm{NaCl}$ substrates heated to room temperature and $50^{\circ} \mathrm{C}$, respectively. At room temperature the TiN film is crystalline and highly textured. At $50^{\circ} \mathrm{C}$ the SAED patterns showed only discrete reflections which could be indexed to TiN, indicating that film growth is epitaxial on $\mathrm{NaCl}$ at this temperature. SAED patterns obtained from films deposited at higher temperatures all appeared identical to Fig. 4b. This result may at first be surprising 
because of the large lattice mismatch (29\%) between TiN and $\mathrm{NaCl}\left(\mathrm{a}_{0}=0.564 \mathrm{~nm}\right)$. However, there exists an almost exact 4:3 coincident site lattice (where 4 unit cells of TiN match with three unit cells of $\mathrm{NaCl}$ ) with a mismatch of only $0.2 \%$. Thus as in the case of $\mathrm{TiN}$ growth on $\mathrm{Si}$, domain matching appears to be an important feature in determining epitaxial growth in systems with large lattice mismatches [4]. The structural similarity between $\mathrm{NaCl}$ and $\mathrm{TiN}$ may also favor epitaxy with the TiN molecules occupying cation and anion sites on the $\mathrm{NaCl}(100)$ surface. This type of "auto-epitaxial" system can lead to epitaxial growth at low temperatures despite large lattice mismatches [7].

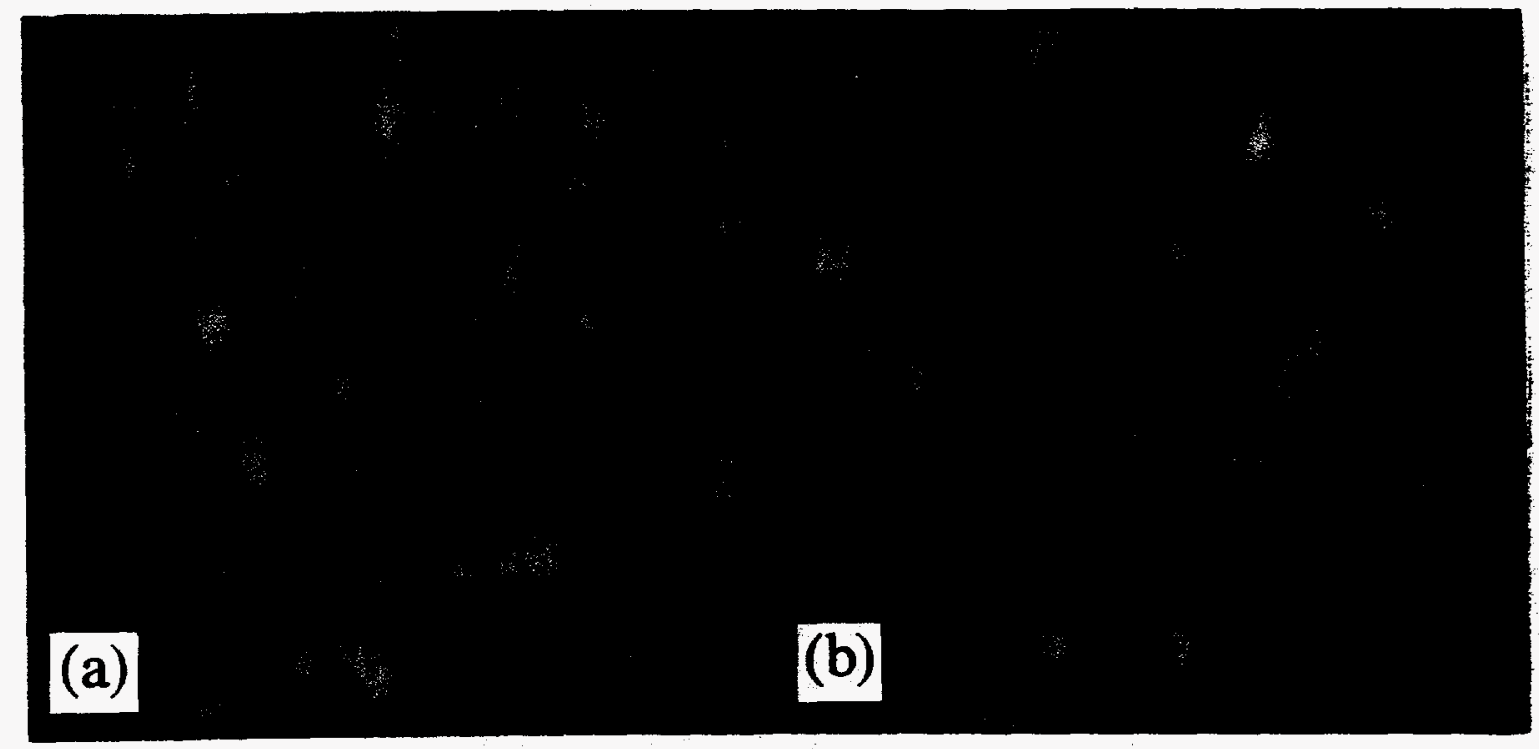

Figure 3. Electron channelling patterns recorded from (a) (100)-oriented Si and (b) a very thin TiN film deposited onto $\mathrm{Si}$ at a substrate temperature of $300^{\circ} \mathrm{C}$.
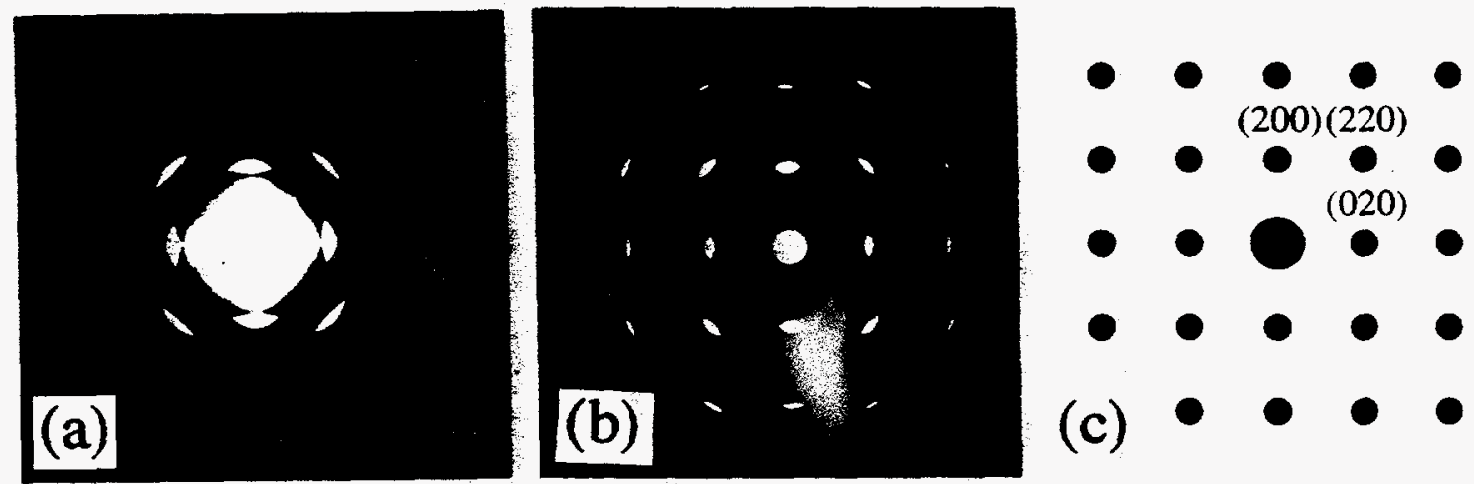

Figure 4. Selected area electron diffraction patterns from TiN thin films deposited onto $\mathrm{NaCl}$ substrates heated to (a) room temperature and (b) $50^{\circ} \mathrm{C}$. An indexed schematic is shown in (c).

Figure 5 shows a PEELS spectrum obtained from a TiN film deposited at room temperature. The absolute composition of the films could not be determined from these spectra because there is some overlap between the Ti- $\mathrm{L}_{1}$ edge which occurs at $564 \mathrm{eV}$ and the O-K edge at $532 \mathrm{eV}$. Absolute composition determination would require multiple-least-squares analysis of second difference spectra using appropriate standards. However, relative changes in film 
composition were noted from examining the jump ratios of the $\mathrm{N}-\mathrm{K}, \mathrm{Ti}-\mathrm{L}_{2,3}$, and $\mathrm{O}-\mathrm{K}$ edges. The jump ratio is the ratio of the intensity at the edge-onset relative to the background intensity immediately before the edge. These ratios indicate that the amount of oxygen incorporated into the TiN films increases and the amount of nitrogen decreases when the film is grown at room temperature rather than $300^{\circ} \mathrm{C}$. Oxygen contamination appears to be a frequent occurrence in TiN films. Several possible sources of this contamination have been identified $[1,3]$.

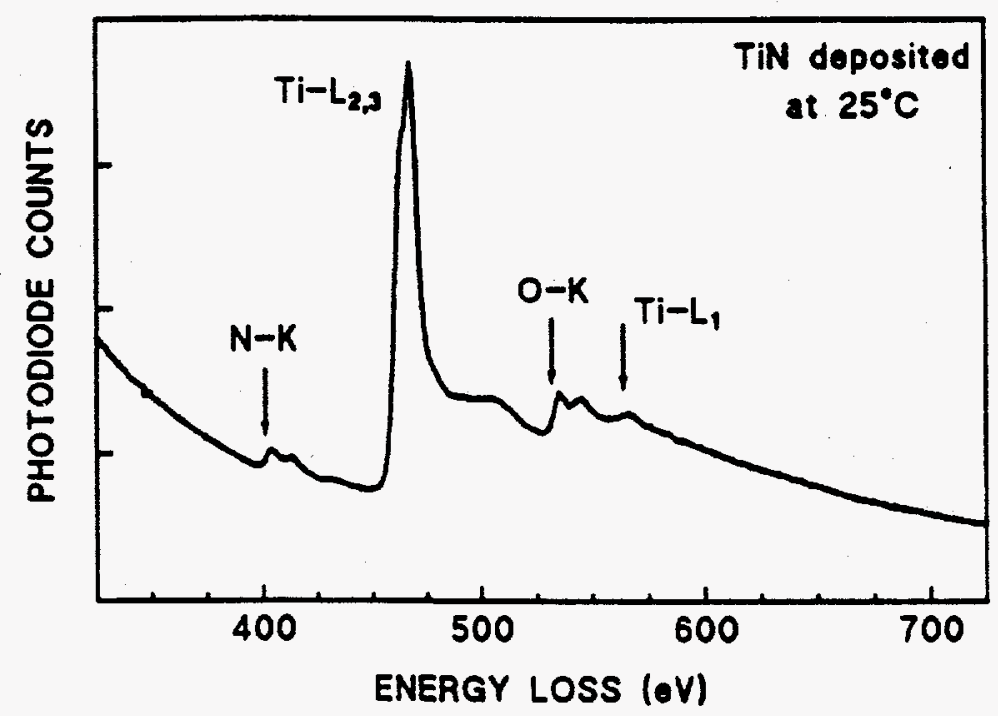

Figure 5. PEELS spectrum of TiN thin film deposited at $25^{\circ} \mathrm{C}$.

\section{CONCLUSION}

In conclusion, extremely smooth oriented $\mathrm{TiN}$ thin films can be grown on single crystal silicon substrates by pulsed-laser deposition at relatively low substrate temperatures. Epitaxial TiN can be formed on $\mathrm{NaCl}$ at a substrate temperature as low as $50^{\circ} \mathrm{C}$.

The work at ORNL was sponsored by the Division of Materials Sciences, US Department of Energy, under contract DE-AC05-84OR21400 with Martin Marietta Energy Systems, Inc., and through the SHaRE Program under contract DE-AC05-76OR00033 with Oak Ridge Associated Universities.

\section{REFERENCES}

1. J.C.S. Kools, C.J.C.M. Nillesen, S.H. Brongersma, E. van de Riet, and J. Dieleman, J. Vac. Sci. Technol. A 10, 1809 (1992)

2. S. Altshulin, A. Rosen, and J. Zahavi, J. Mater. Sci. 28, 3749 (1993)

3. : O. Auciello, T. Barnes, S. Chevacharoenkul, A.F. Schreiner, and G.F. McGuire, Thin Solid Films 181, 73 (1989)

4. J. Narayan, P. Tiwari, X. Chen, J. Singh, R. Chowdhury, and T. Zheleva, Appl. Phys. Lett. 61, 1290 (1992)

5. L.E. Toth, Transition Metal Carbides and Nitrides, Academic Press, New York (1971)

6. U.C. Oh, J.H. Je, and J.Y. Lee, J. Mater. Res. 10, 634 (1995)

7. B. Lewis and D.J. Stirland, J. Cryst. Growth 3,4, 200 (1968) 